\title{
QUANTUM COHERENT SPACES AND LINEAR LOGIC
}

\author{
SteFAno BARATELla ${ }^{1}$
}

\begin{abstract}
Quantum Coherent Spaces were introduced by Girard as a quantum framework where to interpret the exponential-free fragment of Linear Logic. Aim of this paper is to extend Girard's interpretation to a subsystem of linear logic with bounded exponentials. We provide deduction rules for the bounded exponentials and, correspondingly, we introduce the novel notion of bounded exponentials of Quantum Coherent Spaces. We show that the latter provide a categorical model of our system. In order to do that, we first study properties of the category of Quantum Coherent Spaces.
\end{abstract}

Mathematics Subject Classification. 68Q55, 03F52.

\section{INTRODUCTION}

Quantum Coherent Spaces (briefly: QCS's) were introduced by Girard as a framework for interpreting logic into a quantum setting, thus moving in the opposite direction with respect to the attempts of capturing "quantum" by "logic" made by quantum logic. Girard's goal is not the impossible reconciliation of the "separate lives" of logic and quantum physics. More modestly, he wants to investigate how a nondeterministic phenomenon like quantum measurement can be used to model a logical system. See [5] and [6] for the detailed motivations. Actually, QCS's provide an interpretation of the the exponential-free fragment of Linear Logic $([5])$.

Aim of this paper is to extend Girard's work to obtain a categorical model of a subsystem of linear logic with bounded exponentials. Such subsystem turns out to be a simplified version of the Bounded Linear Logic introduced in [7]. So we stress that, even if we will quite extensively work in a categorical setting and we will eventually investigate the computational complexity of a normalization

Keywords and phrases. Quantum coherent spaces, linear logic, bounded exponentials, denotational semantics, normalization.

1 Dipartimento di Matematica, Università di Trento, via Sommarive 14, 38050 Povo, Italy.

baratell@science.unitn.it; stefano.baratella@unitn.it 
procedure, this work is neither about the categorical properties of QCS's nor about the (implicit) computational complexity of yet another logical system.

We also point out from the very beginning that QCS's are confined to the finite dimensional case, hence they cannot model "infinitary connectives" like the exponentials. This limitation is due to the fact that, for operators on infinite dimensional Hilbert spaces, the notion of trace is problematic. Indeed, the discussion in [5], Section 17.6.1 seems to suggest that there is no way of circumventing the limitation due to finite dimension. Therefore, the best we can do is to extend Girard's work to cover some kind of bounded exponentials.

QCS's are closely related to spaces of density matrices. They form a *-autonomous category, that we will denote by QCS. There is a natural notion of morphism between QCS's. Indeed morphisms between QCS's turn out to be positive maps with respect to a suitable preorder defined on the linear span of a QCS (see Sect. 3), hence not the analogue of completely positive maps between spaces of density matrices.

Being *-autonomous, QCS has enough structure to model higher-order linear language features. Nevertheless, in [12] Selinger shows that his presentation of QCS's is not adequate for modelling higher-order quantum computation. This is a good point to note that Selinger's presentation differs in many aspects from ours. In spite of the different perspective from which QCS's are viewed, a partial correspondence between the two presentations can be established (see [12]), but with some relevant differences. For instance, morphisms in Selinger's presentation have to be treated as equivalence classes of morphisms in the sense of this paper. Thus the results claimed in [12] do not immediately apply to the current setting (in this regard, see the description of the content of Sect. 3 below).

In spite of the already mentioned and of further limitations (see below), we believe that QCS is interesting in its own and that its capability of modelling bounded exponentials worths being investigated.

In Sections 2 and 3 we present a number of results about QCS's. Most of them will be used in following sections. Some are interesting in their own and seem to be novel.

In Section 3, for sake of completeness we prove quite in detail that QCS is *-autonomous. We remark that QCS is also an isoMIX category, in the sense of Cockett and Seely ([4]). Recall that the categorical formulation of QCS in the framework of normed cones given in [12] is *-autonomous, has products and coproducts. Furthermore, the author claims the equivalence of the two formulations. Quite surprisingly, we show that QCS lacks a "natural" structure of Cartesian category: a disappointing fact from a categorical viewpoint, that seems to prevent further extensions of our work along the line, for instance, of [11], Sections 5,6. Moreover, such negative result casts a doubt on the categorical equivalence of the current formulation and the one in [12]. It should be added that, although "\&" is not a product in the category QCS, it is nevertheless a weak product.

Although some of the results proved in the first part of this paper are implicitly hinted in [5], Chapter 17 and some others are well-known, we believe that our 
work is of interest also as a complement and a completion of the above-mentioned reference.

In Section 4, we show that QCS provides a denotational semantics for the exponential-free multiplicative fragment MLL of Linear Logic. Actually, this follows immediately from the category being $*$-autonomous. Nevertheless, here we provide a complete proof of this result, to show the import of the results proved in the previous sections and to provide an explicit description of the denotational process. As expected, formulas will be interpreted as QCS's and proofs as some positive maps between QCS's. A reader familiar with coherent spaces and with their being a model of linear logic (see [5], for instance) will recognize here a very similar situation.

In Section 5 we define the bounded exponentials of QCS's. Such definition is based on the novel notion of symmetric tensor power of QCS's, which, in turn, is an analogue in the QCS's setting of the symmetric tensor product of Hilbert spaces, a central notion in quantum physics. (The reader is invited to examine the technical results that motivate the previous definitions). We do not explore here the property of functoriality of the bounded exponentials and which universal properties their construction satisfies.

We also introduce the bounded exponentials of formulas. We extend MLL with deduction rules for the bounded exponentials to form the system B!LL, which, basically, is a subsystem of Bounded Linear Logic. We show that QCS provides a denotational semantics to B!LL proofs as well. Eventually, in Section 6 we show that B!LL is not badly behaved from a proof-theoretic viewpoint. We outline a normalization procedure for B!LL proofs that is based on an analogous result obtained in [7]. As with Bounded Linear Logic, the normalization procedure does not lead to full cut-elimination, due to the presence of certain "bad" cuts. The complexity of the normalization is cubic in the weight (a quite natural complexity measure of proofs), an evidence that B!LL is also computationally well-behaved with respect to normalization.

We briefly discuss the relevant question whether a suitable extension of B!LL to second order has a complexity import. This would further motivate the study of B!LL. We conjecture that a suitable second order extension of B!LL provides yet another implicit characterization of polytime, but we do not provide any technical detail because this goes beyond the scope of this paper.

We assume that the reader is familiar with very basic functional analysis and operator theory. Useful references are [9] and [13] respectively, but any introductory book to those subjects would do.

\section{Preliminaries}

We denote by the letters $V, W, X, Y, Z$ finite dimensional Hilbert spaces on the field $\mathbb{C}$ of complex numbers. We always work in a finite dimensional setting.

As customary, we denote by $V \otimes W$ the tensor product of the Hilbert spaces $V$ and $W$. Although the symbol $\otimes$ will be used later with a different meaning, the 
context will prevent any ambiguity. We recall that $V \otimes W$ is a Hilbert space with the induced Hermitian product

$$
\left\langle v_{1} \otimes w_{1}, v_{2} \otimes w_{2}\right\rangle=\left\langle v_{1}, v_{2}\right\rangle_{V}\left\langle w_{1}, w_{2}\right\rangle_{W},
$$

where $\left\langle_{-},{ }_{-}\right\rangle_{V}$ and $\left\langle_{-},{ }_{-}\right\rangle_{W}$ are the Hermitian products on $V$ and $W$ respectively.

We denote by $H(X)$ the real vector space of Hermitian operators on the space $X$. If the complex dimension of $X$ is $n$, then the dimension of $H(X)$ is $n^{2}$. Since $H(X) \otimes H(Y)$ is a subspace of $H(X \otimes Y)$ and the two have equal finite dimension, it follows that $H(X \otimes Y)=H(X) \otimes H(Y)$.

We denote by $\operatorname{tr}(f)$ the trace of the operator $f \in H(X)$. The trace induces an inner product $\left\langle_{-},-\right\rangle$on $H(X)$ defined by $\langle f, g\rangle=\operatorname{tr}(f g)$, hence $H(X)$ has a structure of real Euclidean space. We define the trace norm of $f \in H(X)$ as usual: $\|f\|=\langle f, f\rangle^{1 / 2}$.

Note: to define a linear map, very frequently we will define it just on a basis or on a set of generators.

Definition 2.1 (polarity).

(1) Two Hermitians $f, g \in H(X)$ are polar (notation: $f \sim g$ ) if $0 \leq \operatorname{tr}(f g) \leq 1$.

(2) Let $A \subseteq H(X)$. The polar of $A$ is the set

$$
\sim A=\{g \in H(X): g \sim f \text { for all } f \in A\} .
$$

Cyclicity of trace (namely the property that $\operatorname{tr}(f g)=\operatorname{tr}(g f)$ ) implies that the relation $\sim$ is symmetric.

Definition 2.2. The set $A \subseteq H(X)$ is a Quantum Coherent Space (briefly: QCS) if $A=\sim \sim A$

Examples of QCS's are given in [5].

The following are easily proved for all $A \subseteq H(X)$ :

(1) $A \subseteq \sim \sim A$;

(2) $\sim A=\sim \sim \sim A$;

(3) $\sim \sim A$ is the least QCS containing $A$.

From 2. above it follows that $A \subseteq H(X)$ is a QCS if and only if $A=\sim B$ for some $B \subseteq H(X)$.

In [5], Girard proves the following useful characterization of QCS's:

Theorem 2.3 (Bipolar Theorem). Let $C \subseteq H(X)$. The set $C$ is a $Q C S$ if and only if

(1) $0 \in C$;

(2) $C$ is closed and convex;

(3) if $n f \in C$ for all $n \in \mathbb{N}$ then $-f \in C$;

(4) if $f, g \in C$ and $\lambda, \mu$ are nonnegative real numbers such that $\lambda f+\mu g \in C$ then $\lambda f \in C$. 
We will use the letters $C, D, E$ (possibly indexed) to denote QCS's.

In [5], Girard adds extra structure to a QCS $C \subseteq H(X)$. He defines on the linear span Fin $\mathrm{C}$ of $C$ a seminorm $\|\cdot\|_{C}$ and a preorder $\preccurlyeq_{C}$ as follows:

$$
\begin{gathered}
\|f\|_{C}=\sup \{|\operatorname{tr}(f g)|: g \in \sim C\} ; \\
f \preccurlyeq C g \Leftrightarrow \operatorname{tr}(f h) \leq \operatorname{tr}(g h) \text { for all } h \in \sim C .
\end{gathered}
$$

It is straightforward to check that $C=\left\{f \in \mathrm{Fin}_{\mathrm{C}}: 0 \preccurlyeq_{\mathrm{C}} \mathrm{f}\right.$ and $\left.\|\mathrm{f}\|_{\mathrm{C}} \leq 1\right\}$ and so the positive cone $C^{+}=\bigcup_{r \in \mathbb{R}>0} r C=\bigcup_{n \in \mathbb{N}} n C$ of $C$ is the set of $\preccurlyeq C$-positive elements of Fin $\mathrm{C}$, where the rightmost equality holds by Theorem 2.3.4.

Therefore one may regard a QCS $\mathrm{C}$ as the set of positive elements in the unit ball of the semi-normed preordered space Fin $_{C}$ (with respect to $\|\cdot\|_{C}$ and $\preccurlyeq_{C}$ ). This remark will be useful later when defining a suitable family of maps between QCS's.

The Bipolar Theorem provides a large class of QCS's: for all $X$, each linear subspace $S$ of $H(X)$ is a QCS. In a sense, those are the less interesting QCS's: for $S$ as above, $\sim S$ is the orthogonal space $S^{\perp}$ to $S$ and therefore $\|f\|_{S}=0$ and $f \preccurlyeq s g$ for all $f, g \in S$.

We denote by [·] the linear isomorphism between $H(X \otimes Y)$ and the space $\operatorname{hom}(H(X), H(Y))$ of $\mathbb{R}$-linear maps from $H(X)$ to $H(Y)$ implicitly defined is as follows:

$$
\langle([F] f)(y), z\rangle=\operatorname{tr}\left(F \circ\left(f \otimes y z^{*}\right)\right) \quad \text { for } F \in H(X \otimes Y), f \in H(X) \text { and } y, z \in Y,
$$

where $y z^{*}: w \mapsto\langle w, z\rangle y$ for all $w \in Y$.

We will extensively use such an isomorphism in the sequel. Its most important property is the following: for all $F \in H(X \otimes Y), f \in H(X), g \in$ $H(Y)$

$$
\operatorname{tr}(([F] f) \circ g)=\operatorname{tr}(F \circ(f \otimes g))
$$

Given the QCS's $C \subseteq H(X)$ and $D \subseteq H(Y)$, Girard defines the following QCS:

$$
\begin{aligned}
& C \multimap D=\{F \in H(X \otimes Y):[F] c \in D \text { for all } c \in C\} ; \\
& C \otimes D=\sim \sim\{c \otimes d: c \in C \text { and } d \in D\} ; \\
& C \text { ช } D=(\sim C) \multimap D .
\end{aligned}
$$

In order to prove that $C \multimap D$ is a QCS it suffices to show that

$$
C \multimap D=\sim\{c \otimes d: c \in C \text { and } d \in \sim D\}
$$

Equality (3) follows at once from (2).

Note that the previous definitions can be given for arbitrary sets $C \subseteq H(X)$ and $D \subseteq H(Y)$.

If $C$ is a linear subspace of $H(X)$ then $\sim C=C^{\perp}$, where $C^{\perp}$ is the orthogonal of $C$. Moreover, if we denote by $E$ the algebraic tensor product of the linear spaces $C \subseteq H(X)$ and $D \subseteq H(Y)$, then $E^{\perp}=\sim E=\sim\{f \otimes g: f \in C$ and $g \in D\}$. Since 
by finite dimensionality $E=E^{\perp \perp}$, it follows that $E=\sim \sim\{f \otimes g: f \in C$ and $g \in$ $D\}$. Hence $C \otimes D$ is just the algebraic tensor product of the linear spaces $C$ and $D$, as expected.

Proposition 2.4. Let $C, D$ be $Q C S$ 's. Then $C$ > $D=\sim(\sim C \otimes \sim D)$.

Proof. From (3) we get:

$$
F \in(C \ngtr D) \Leftrightarrow 0 \leq \operatorname{tr}(F \circ(c \otimes d)) \leq 1 \text { for all } c \in \sim C \text { and all } d \in \sim D
$$

from which the conclusion follows immediately.

\section{The CATEgory QCS}

In this section we introduce the category QCS of Quantum Coherent Spaces and we show that it is $*$-autonomous. The same result holds for the different formulation of QCS's given in [12] (see the comments in Sect. 1). Here we provide a quite detailed proof that QCS is *-autonomous, mostly for sake of completeness.

The objects of the category QCS are the QCS's. Let $C \subseteq H(X)$ and $D \subseteq H(Y)$ be QCS's. The morphisms between $C$ and $D$ are the elements of $C \multimap D$.

Next we define the composition of morphisms: let $F \in C \multimap D$ and $G \in D \multimap E$. We define $G \circ F$ as follows:

$$
[G \circ F]=[G] \circ[F]
$$

where [.] is the linear mapping implicitly defined in (1). The composition of morphisms is clearly associative. Moreover, for each $D \subseteq H(X)$ there exists a morphism $\operatorname{id}_{D} \in H(X \otimes X)$ that is an identity with respect to composition of morphisms. From the definition of composition it must be that $\left[\mathrm{i} d_{D}\right]=\mathrm{id}_{H(X)}$. Therefore $\operatorname{id}_{D}=$ twist, where twist $\in H(X \otimes X)$ is (the linear extension of the map) defined by

$$
\text { twist : } f \otimes g \mapsto g \otimes f .
$$

For, $[$ twist $]=\operatorname{id}_{H(X)}($ see $[5])$.

Once we have defined composition of morphisms and identities, the notion of isomorphism is the standard (category-theoretic) one. We shall write $C \simeq D$ to say that $C$ and $D$ are isomorphic QCS's.

Proposition 3.1. Let $C \subseteq H(X)$ and $D \subseteq H(Y)$ be $Q C S$ 's. The following are equivalent:

(1) $C \simeq D$;

(2) there exists a linear isomorphism $L: H(X) \rightarrow H(Y)$ such that $L(C)=D$.

Proof. $(1) \Rightarrow(2)$ is completely straightforward.

As for $(2) \Rightarrow(1)$, let $L$ be as in $(2)$ and let $T=L^{-1}$. Then $[T]^{-1} \circ[L]^{-1}=\mathrm{id}_{C}$ and $[L]^{-1} \circ[T]^{-1}=\mathrm{id}_{D}$. 
Let $F \in C \multimap D$. Then $\|[F](g)\|_{D} \leq\|g\|_{C}$ and

$$
\left.g \preccurlyeq_{C} h \Rightarrow[F](g) \preccurlyeq_{D}[F](h)\right) \text { for all } g, h \in \operatorname{Fin}_{C} .
$$

If $F$ is an isomorphism then $\|[F](g)\|_{D}=\|g\|_{C}$ and

$$
g \preccurlyeq_{C} h \Leftrightarrow[F](g) \preccurlyeq_{D}[F](h) \text { for all } g, h \in \operatorname{Fin}_{C} .
$$

Here is a sketch of proof of the latter equivalence:

$$
g \preccurlyeq_{C} h \Leftrightarrow h-g \in C^{+} \Leftrightarrow[F](h)-[F](g) \in D^{+} \Leftrightarrow[F](g) \preccurlyeq_{D}[F](h) .
$$

Therefore the isomorphism $F$ induces seminorm - and order - preserving linear isomorphism between Fin $_{C}$ and Fin $_{D}$ that is compatible with the seminorms and the preorders defined on $C$ and $D$, as expected.

Conversely, if $L$ is a seminorm - and order - preserving linear isomorphism between $\mathrm{Fin}_{C}$ and $\mathrm{Fin}_{D}$, it is not always the case that $L$ induces an isomorphism between $C$ and $D$ : this is the case exactly when $X$ and $Y$ have the same dimension. We will see later that, from our definition of isomorphism, it follows that

$$
C \simeq D \Rightarrow \sim C \simeq \sim D, \quad \text { for all QCS's } C, D .
$$

The previous property does not hold in general if we define an isomorphism between $C$ and $D$ as a linear isomorphism $L$ between Fin $_{C}$ and $\operatorname{Fin}_{D}$ that is compatible with seminorms and preorders (equivalently: such that $L(C)=D$ ).

In the rest of this section, we will first prove the existence of a number of useful (although expected) isomorphisms among QCS's. Then we will sketch a proof that QCS is a $*$-autonomous category, as the category of coherent spaces is (see [3]). We prefer not to immediately start proving that QCS is $*$-autonomous in order to get more familiarity with QCS's. This choice will involve a certain amount of repetition later on.

Proposition 3.2. In $\mathbf{Q C S}$ there is an object $\mathbf{1}$ such that $C \otimes \mathbf{1} \simeq C$ and $\mathbf{1} \otimes C \simeq C$ for all QCS's $C$.

Proof. Let $\mathbf{1}=\{g \in H(\mathbb{C}): 0 \leq \operatorname{tr}(g) \leq 1\}$, so $\mathbf{1}$ is isomorphic to the real interval $[0,1]$. It is clear that the Hermitian $\rho_{C}$ defined by $\left[\rho_{C}\right]: f \otimes \operatorname{id}_{\mathbb{C}} \mapsto f$ yields an isomorphism between $C \otimes \mathbf{1}$ and $C$. Similarly for the other isomorphism $\lambda_{C}$. fact:

We denote by $T^{*}$ the adjoint of an operator $T$. We recall a fairly elementary

Proposition 3.3. Let $T: V \rightarrow W$ be a linear isometric isomorphism of finite dimensional complex Hilbert spaces. Let $\operatorname{End}(V), \operatorname{End}(W)$ be the spaces of linear endomorphisms of $V, W$ respectively. Then the map $\bar{T}: \operatorname{End}(V) \rightarrow \operatorname{End}(W)$ defined by $\bar{T}(f)=T f T^{*}$ is a linear isomorphism that satisfies the following properties:

(1) $\bar{T}(f \circ g)=\bar{T}(f) \circ \bar{T}(g)$ for all $f, g \in \operatorname{End}(V)$; 
(2) $\operatorname{tr}(\bar{T}(f))=\operatorname{tr}(f)$ for all $f \in \operatorname{End}(V)$;

(3) the restriction of $\bar{T}$ to $H(V)$ is a $\mathbb{R}$-linear isomorphism onto $H(W)$ with the property that $\bar{T}(\sim A)=\sim \bar{T}(A)$ for all $A \subseteq H(V)$.

Proof. The proofs of (1) and (2) are straightforward.

As for the first part of (3), in order to prove that, $\bar{T}(f)$ is in $H(W)$ when $f \in H(V)$, it suffices to show that $\langle\bar{T}(f)(T(x)), y\rangle=\langle T(x), \bar{T}(f)(y)$, for all $x \in V$ and all $y \in W$. Note that

$$
\langle\bar{T}(f)(T(x)), y\rangle=\langle T f(x), y\rangle=\left\langle f(x), T^{*}(y)\right\rangle=\left\langle x, f T^{*}(y)\right\rangle=\left\langle T(x), T f T^{*}(y)\right\rangle,
$$

as required.

The second part of (3) is also straightforward.

We are now ready to prove the following:

Proposition 3.4. Let $C \subseteq H(X)$ and $D \subseteq H(Y)$ be $Q C S$ 's. Then $C \otimes D \simeq D \otimes C$.

Proof. Let $T: X \otimes Y \rightarrow Y \otimes X$ be the linear isomorphism defined by $x \otimes y \mapsto y \otimes x$. By Proposition 3.3, $T$ induces a linear isomorphism $\bar{T}: H(X \otimes Y) \rightarrow H(Y \otimes X)$ with the property that $\bar{T}(C \otimes D)=\sim \sim \bar{T}(\{c \otimes d: c \in C, d \in D\})$. Since $\bar{T}(f \otimes g)=$ $g \otimes f$ for all $f \in H(X), g \in H(Y)$, it follows that $\bar{T}(C \otimes D)=D \otimes C$. By Proposition 3.1 we finally get $C \otimes D \simeq D \otimes C$.

Each $*$-autonomous category is equipped with a (not necessarily unique) dualizing object $\perp$, satisfying certain properties (see [3]). It is straightforward to prove that the object $\perp=\mathbf{1}$ behaves as a dualizing object in QCS (see Prop. 3.22). Notice that $\sim \perp=\perp$.

The following holds:

Proposition 3.5. Let $C \subseteq H(X)$ be a $Q C S$. Then $\sim C \simeq(C \multimap \perp)$.

Proof. The elements of $H(X \otimes \mathbb{C})$ are of the form $f \otimes \operatorname{id}_{\mathbb{C}}$, with $f \in H(X)$. By applying (2) and the properties of trace, for all $f \in H(X)$ we get the following chain of equivalences:

$$
\begin{aligned}
f \otimes \operatorname{id}_{\mathbb{C}} \in C \multimap \perp & \Leftrightarrow 0 \leq \operatorname{tr}\left(\left[f \otimes \mathrm{id}_{\mathbb{C}}\right] g\right) \leq 1 \quad \text { for all } g \in C \\
& \Leftrightarrow 0 \leq \operatorname{tr}\left(\left(\left[f \otimes \mathrm{id}_{\mathbb{C}}\right] g\right) \otimes i d_{\mathbb{C}}\right) \leq 1 \quad \text { for all } g \in C \\
& \Leftrightarrow 0 \leq \operatorname{tr}\left((f \otimes \mathrm{id} \mathbb{C}) \circ\left(g \otimes i d_{\mathbb{C}}\right)\right) \leq 1 \quad \text { for all } g \in C \\
& \Leftrightarrow 0 \leq \operatorname{tr}(f g) \leq 1 \quad \text { for all } g \in C \\
& \Leftrightarrow f \in \sim C .
\end{aligned}
$$

The conclusion follows by applying Proposition 3.1 to the linear isomorphism $f \mapsto$ $f \otimes \operatorname{id}_{\mathbb{C}}$ between $H(X)$ and $H(X \otimes \mathbb{C})$.

Proposition 3.6. Let $C \subseteq H(X)$ and $D \subseteq H(Y)$ be QCS's. Then $C \multimap D \simeq$ $\sim D \multimap \sim C$. 
Proof. By Proposition 3.1 it suffices to prove that $T(C \multimap D)=\sim D \multimap \sim C$ for some linear isomorphism $T: H(X \otimes Y) \rightarrow H(Y \otimes X)$. From (3) we get $\sim D \multimap \sim C=\sim\{d \otimes c: d \in \sim D, c \in C\}$ and we can proceed as in the proof of Proposition 3.4.

Corollary 3.7. $C \ngtr 8 D \simeq D \ngtr C$ for all $Q C S$ 's $C, D$.

Proposition 3.8. Let $C \subseteq H(X), D \subseteq H(Y)$ and $E \subseteq H(Z)$ be QCS's with $C \simeq D$. Then $C \multimap E \simeq D \multimap E$.

Proof. Given a linear isomorphism $L: H(X) \rightarrow H(Y)$ such that $L(C)=D$, we define a linear isomorphism $K: H(Y \otimes Z) \rightarrow H(X \otimes Z)$ such that $K(D \multimap E)=$ $C \multimap E$, as follows:

$$
[K(F)]=[F] \circ L .
$$

Corollary 3.9. Let $C, D$ be isomorphic QCS's. Then $\sim C \simeq \sim D$.

Proof. Straightforward from Propositions 3.5 and 3.8.

Proposition 3.10. 8 and $\otimes$ are associative up to isomorphism.

Proof. Let $C \subseteq H(X), D \subseteq H(Y), E \subseteq H(Z)$ be QCS's. Then

$$
\begin{gathered}
(C \ngtr D) \ngtr E \simeq E \ngtr(C \ngtr D)=\sim E \multimap(\sim C \multimap D) \text { and } \\
C \ngtr 8(D \ngtr E) \simeq \sim C \multimap(\sim E \multimap D) .
\end{gathered}
$$

Therefore it suffices to show that there exists a linear isomorphism

$$
L: H(Z \otimes(X \otimes Y)) \rightarrow H(X \otimes(Z \otimes Y))
$$

such that $L(\sim E \multimap(\sim C \multimap D))=\sim C \multimap(\sim E \multimap D)$. Given $F \in H(Z \otimes(X \otimes$ $Y)$ ), we define $L(F)$ as follows:

$$
[[L(F)](f)](h)=[[F](h)](f),
$$

for $f \in H(X)$ and $h \in H(Z)$. It is easy to check that $L$ has the required properties.

As for the associativity of $\otimes$, one repeatedly uses $C \otimes D=\sim(\sim C \not \gamma \sim D)$.

Part of the proof of the the basic adjunction isomorphism of $*$-autonomous categories is now straightforward:

Proposition 3.11. $C \otimes D \multimap E \simeq D \multimap(C \multimap E)$ for all $Q C S$ 's $C, D, E$.

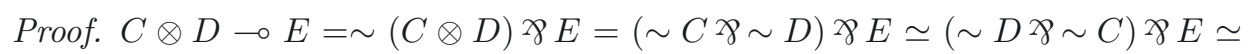
$\sim D \ngtr(\sim C \not \gamma \sim E)=D \multimap(C \multimap E)$.

The morphism $F \in(C \otimes D \multimap E) \multimap(D \multimap(C \multimap E))$ defined by

$$
[[[F](g)](u)](v)=[g](v \otimes u)
$$

explicitly provides an isomorphism. 
All the identities among linear connectives can be proved true (at least up to isomorphism) in the framework of QCS's.

Remark 3.12. Notice that all the isomorphisms established so far are canonical, in the sense that they do not depend on a basis choice for spaces of Hermitians. This is also true of all the isomorphisms that will be established in the sequel.

We prove a result that will be useful in the sequel. (Recall that $\langle f, g\rangle=\operatorname{tr}(f g)$, for $f, g$ Hermitian operators.)

Lemma 3.13. Let $C_{1}, \ldots, C_{n}$ be $Q C S$ 's and let $K$ be a Hermitian such that

$0 \leq\left\langle K, f_{1} \otimes\left(f_{2} \otimes \cdots \otimes\left(f_{n-1} \otimes f_{n}\right) \ldots\right)\right\rangle \leq 1$ for all $1 \leq i \leq n$ and all $f_{i} \in \sim C_{i}$.

Then $K \in C_{1} \&\left(C_{2} \& \ldots\right.$ \& $\left.\left(C_{n-1} \& C_{n}\right) \ldots\right)$.

Proof. If $n=2$ then, from (2), we get $0 \leq\left\langle[K] f_{1}, f_{2}\right\rangle \leq 1$ for all $f_{1} \in \sim C_{1}$ and all $f_{2} \in \sim C_{2}$. Hence $[K] f_{1} \in C_{2}$ for all $f_{1} \in \sim C_{1}$ and so $K \in \sim C_{1} \multimap C_{2}=C_{1}$ > $C_{2}$.

For $n>2$, repeatedly apply (2).

Note that associativity of 28 allows a bracket-free formulation of Lemma 3.13, where in both $f_{1} \otimes \cdots \otimes f_{n}$ and $C_{1}>8 \ldots \gamma C_{n}$ the missing brackets are understood to be placed in the same way:

Lemma 3.14. Let $C_{1}, \ldots, C_{n}$ be $Q C S$ 's. The following are equivalent for a Hermitian $K$ :

(1) $\left.0 \leq\left\langle K, f_{1} \otimes \cdots \otimes f_{n}\right)\right\rangle \leq 1$ for all $f_{i} \in \sim C_{i}$ and all $1 \leq i \leq n$;

(2) $K \in C_{1} \not \ldots$ \& $C_{n}$.

Proof. For $(2) \Rightarrow(1)$, use $C_{1} \not \supset \ldots \not 8 C_{n}=\sim\left(\sim C_{1} \otimes \cdots \otimes \sim C_{n}\right)$.

Proposition 3.15. Let $A \subseteq H(X)$ and $B \subseteq H(Y)$ be arbitrary sets. Then $A \multimap(\sim B)=\sim\{a \otimes b: a \in \bar{A}$ and $b \in B\}$.

Proof. Straightforward.

Proposition 3.16. Let $A \subseteq H(X)$ and $B \subseteq H(Y)$ be arbitrary sets. Then $A \otimes B=A \otimes \sim \sim B$.

Proof. By Proposition 3.15 we get

$$
\begin{aligned}
A \otimes B & =\sim(A \multimap \sim B)=\sim(A \multimap \sim \sim \sim B)=\sim \sim\{a \otimes c: a \in A \text { and } c \in \sim \sim B\} \\
& =A \otimes \sim \sim B .
\end{aligned}
$$

Corollary 3.17. Let $A \subseteq H(X)$ and $B \subseteq H(Y)$ be arbitrary sets. Then $A \otimes B$ $=\sim \sim A \otimes \sim \sim B$.

Proof. Follows from Proposition 3.16 by inspecting the behaviour of the isomorphism defined in the proof of Proposition 3.4. 
In the rest of this section we will sketch a proof that QCS is a *-autonomous category. We recall that a $*$-autonomous category is a symmetric monoidal closed category equipped a dualizing object (see [8] and [10] for the definitions). We borrow the notation from [8], Chapter VII.

Proposition 3.18. QCS is monoidal.

Proof. We first show that QCS is equipped with a bifunctor

$$
\otimes: \text { QCS } \times \text { QCS } \rightarrow \text { QCS } .
$$

The definition of $\otimes$ on pairs of coherent spaces is clear. Let us define $\otimes$ on pairs of morphisms.

Let $C_{i} \subseteq H\left(X_{i}\right)$ and $D_{i} \subseteq H\left(Y_{j}\right)$, for $1 \leq i, j \leq 2$, be QCS's. Given $f \in C_{1} \multimap$ $D_{1}$ and $g \in C_{2} \multimap D_{2}$, we define $f \otimes g \in C_{1} \otimes C_{2} \multimap D_{1} \otimes D_{2}$ as follows:

$$
[f \otimes g](u \otimes v)=[f](u) \otimes[g](v) \quad \text { for all } u \in H\left(X_{1}\right) \text { and } v \in H\left(X_{2}\right) .
$$

We prove that $f \otimes g \in C_{1} \otimes C_{2} \multimap D_{1} \otimes D_{2}=\left(\sim C_{1} \ngtr \sim \sim C_{2}\right) \ngtr\left(D_{1} \otimes D_{2}\right)$. By Lemma 3.14 it suffices to show that

$$
0 \leq\langle f \otimes g,(u \otimes v) \otimes w\rangle \leq 1 \text { for all } u \in C_{1}, v \in C_{2} \text { and } w \in \sim\left(D_{1} \otimes D_{2}\right) .
$$

By (2), the previous condition is equivalent to $0 \leq\langle[f \otimes g](u \otimes v), w\rangle \leq 1$, and the latter is satisfied just by definition of $f \otimes g$.

It is easy to prove that the functor $\otimes$ preserves identities and composition of morphisms.

The next step is to check that tensor product of QCS's is associative up to a natural isomorphism $\alpha_{C, D, E} \in(C \otimes D) \otimes E \multimap C \otimes(D \otimes E)$. Note that from Proposition 3.10 we already know associativity of the tensor product of QCS's.

The definition of $\alpha_{C, D, E}$ is clear:

$$
\left[\alpha_{C, D, E}\right]((u \otimes v) \otimes w)=u \otimes(v \otimes w) .
$$

In order to check that $\alpha_{C, D, E}$ is in $(C \otimes D) \otimes E \multimap C \otimes(D \otimes E)$, proceed as above. It is straightforward to prove that $\alpha_{C, D, E}$ is an isomorphism and its naturality (in a category-theoretic sense) is just a matter of tedious calculations.

Moreover, easy "diagram chase" shows that the isomorphisms $\rho$ and $\lambda$ defined in Proposition 3.2 are natural and that the pentagonal and triangular diagrams (see [8]) commute.

This finishes the proof that QCS is a monoidal category.

Remark 3.19. The definition of the natural isomorphism $\alpha_{C, D, E}$ in the proof of Proposition 3.18 is uniform, namely it does not depend on $C, D, E$, but just on the relevant spaces of Hermitians. So we may write $\alpha_{X, Y, Z}$ for $\alpha_{C, D, E}$, if $C \subseteq H(X), D \subseteq H(Y)$ and $E \subseteq H(Z)$. We shall use this property later on. 
Proposition 3.20. QCS is symmetric.

Proof. We have already implicitly shown (see Prop. 2.4 and Cor. 3.7) that the tensor product of coherent spaces is commutative up to isomorphism. Indeed, for all QCS's $C, D$, the morphism $\gamma_{C, D}$ defined by $\left[\gamma_{C, D}\right](u \otimes v)=v \otimes u$ belongs to $C \otimes D \multimap D \otimes C$ and is a natural isomorphism that satisfies $\gamma_{C, D}=\gamma_{D, C}^{-1}$. Furthermore, the hexagonal diagrams do commute.

Proposition 3.21. QCS is closed.

Proof. We show that QCS is equipped with a left closed structure (see [10]).

We define the left evaluation morphism eval $C, D \in C \otimes(C \multimap D) \multimap D$ for all coherent spaces $C, D$ as follows:

$$
\left[\operatorname{eval}_{C, D}\right](u \otimes v)=[v](u) .
$$

We require the morphism $e^{2 v a l}{ }_{C, D}$ to satisfy the following universal property: for all QCS's $E$ and all morphisms $f \in C \otimes E \multimap D$ there exists a unique morphism $h \in E \multimap(C \multimap D)$ such that eval ${ }_{C, D} \circ\left(\operatorname{id}_{C} \otimes h\right)=f$. This requirement forces the following definition of $h$ (hence its uniqueness):

$$
[[h](v)](u)=[f](u \otimes v) .
$$

Proposition 3.22. The object $\perp=\mathbf{1}$ is a dualizing object in QCS.

Proof. Let $C$ be a QCS and let $\delta_{C} \in C \multimap((C \multimap \perp) \multimap \perp)$ be the morphism corresponding to $\mathrm{eval}_{C, \perp} \circ \gamma_{C \multimap \perp, C}$ via the isomorphism $(C \multimap \perp) \otimes C \multimap \perp \simeq C \multimap$ $((C \multimap \perp) \multimap \perp)$ that has been defined in Proposition 3.11. We must check (see [3]) that $\delta_{C}$ is a natural isomorphism.

An easy calculation shows that $\delta_{C}$ is defined by $\left[\left[\delta_{C}\right](u)\right](v)=[v](u)$. The property of natural isomorphism follows immediately from the definition.

Finally, we summarize the previous propositions in the following:

\section{Theorem 3.23. QCS is *-autonomous.}

In the next section we shall see an application of the previous theorem.

In the rest of this section we show that the natural candidate for (Cartesian) product in QCS actually fails. We need a preliminary definition. Let $C_{1} \subseteq H\left(X_{1}\right)$ and $C_{2} \subseteq H\left(X_{2}\right)$ be QCS's. We denote by $X_{1} \oplus X_{2}$ the direct sum of $X_{1}$ and $X_{2}$. The inner product on $X_{1} \oplus X_{2}$ is the sum of the inner products of the two summands. Let $\iota_{i}: X_{i} \rightarrow X_{1} \oplus X_{2}$ and $\pi_{i}: X_{1} \oplus X_{2} \rightarrow X_{i}$ be the canonical embedding/projection of/onto $X_{i}$ respectively, $i=1,2$. We let

$$
C_{1} \& C_{2}=\left\{h \in H\left(X_{1} \oplus X_{2}\right): \pi_{i} h \iota_{i} \in C_{i} \text { for } i=1,2\right\} .
$$

The set $C_{1} \& C_{2}$ is easily proved to be a QCS (use the Bipolar Theorem).

Dually, we have $C_{1} \oplus C_{2}=\left\{\lambda c_{1} \oplus(1-\lambda) c_{2}: c_{1} \in C_{1}, c_{2} \in C_{2}, 0 \leq \lambda \leq 1\right\}$. It is easy to show that $\&$ and $\oplus$ are swapped by negation (see [5]). 
A candidate for the product of $C_{1}$ and $C_{2}$ is the triple $\left(C_{1} \& C_{2}, P_{1}, P_{2}\right)$, where $\left[P_{i}\right](f)=\pi_{i} f \iota_{i}$ for $f \in H\left(X_{1} \otimes X_{2}\right)$ and $i=1,2$. By definition of $C_{1} \& C_{2}$ we have at once that $P_{i} \in\left(C_{1} \& C_{2}\right) \multimap C_{i}, i=1,2$.

Let $E \subseteq H(Z)$ be a QCS and let $F \in\left(E \multimap C_{1}\right), G \in\left(E \multimap C_{2}\right)$. Let $H$ be the map defined by $[H](f)=[F](f) \oplus[G](f)$, when $f \in H(Z)$. Clearly, $P_{1} \circ H=F$ and $P_{2} \circ H=G$. Also, $H \in E \multimap\left(C_{1} \& C_{2}\right)$. However, uniqueness of a map with the same properties as $H$ is not granted, as we show below. For notational convenience we identify maps with matrices representing them with respect to fixed basis. Let $0 \neq f \in H(Z)$ and let $\mathcal{B}$ be a basis of $H(Z)$ extending $\{f\}$. Fix map $0 \neq u$ and define $K$ as follows:

$$
[K](g)=\left\{\begin{array}{cc}
\left(\begin{array}{cc}
{[F](g)} & u \\
u^{*} & {[G](g)}
\end{array}\right) & \text { if } g=f ; \\
{[H](g)} & \text { if } g \in \mathcal{B} \backslash\{f\},
\end{array}\right.
$$

where $u^{*}$ is the transpose conjugate of $u$. Then $H \neq K ; K \in E \multimap\left(C_{1} \& C_{2}\right)$; $P_{1} \circ K=F$ and $P_{2} \circ K=G$.

Whether QCS admits finite products remains an open problem, but the above example suggests that products, if existing, might have a quite unnatural structure.

For sure, QCS does not have a terminal object: the zero and the twist map are distinct maps in $C \multimap C$, for all QCS's $C$.

\section{QCS AS A CATEGORICAL MODEL}

In this section we show that the category QCS is a categorical model of the exponential-free multiplicative fragment MLL of linear logic. Actually, this fact follows immediately from QCS being *-autonomous. Here we proceed in an "algebraic" rather than in a categorical manner, meaning by this that we fully exploit the properties of QCS's that have been proved in the previous sections. Also, we briefly discuss the status of QCS as a category adequate for modelling MIX rule.

We associate to each linear formula $\gamma$ a QCS $\llbracket \gamma \rrbracket$, called the denotation of $\gamma$. Indeed it suffices to provide denotations for the propositional letters and for the logical constants. The former are freely chosen. The denotations of constants 1 and $\perp$ are the objects with the same names in the category QCS.

The the denotation of formula $\gamma^{\perp}$ is $\sim \llbracket \gamma \rrbracket$. For each multiplicative binary linear connective $\star$, the denotation of $\gamma \star \delta$ is $\llbracket \gamma \rrbracket \star \llbracket \delta \rrbracket$.

Strictly speaking, the denotation $\llbracket \vdash \gamma_{1}, \ldots, \gamma_{n} \rrbracket$ of sequent $\vdash \gamma_{1}, \ldots, \gamma_{n}$ should

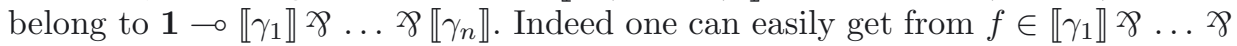
$\llbracket \gamma_{n} \rrbracket$ a Hermitian $F \in \mathbf{1} \multimap \llbracket \gamma_{1} \rrbracket \ngtr \ldots \ngtr \llbracket \gamma_{n} \rrbracket$, simply by letting $[F]: \operatorname{id}_{\mathbb{C}} \mapsto$ f. For this reason we stipulate that $\left.\llbracket \vdash \gamma_{1}, \ldots, \gamma_{n} \rrbracket=\llbracket \gamma_{1} \rrbracket \not \gamma \ldots \curlyvee\right\rceil \gamma_{n} \rrbracket$.

Note that associativity up to isomorphism of $\not 2$ allows us to almost forget about brackets. Formally we stipulate that, in a sequence of $x($ or of $\otimes)$, brackets associate to the left. 
We denote a proof $\Pi$ in MLL by a Hermitian $\llbracket \Pi \rrbracket$ belonging to the denotation of its end-sequent. We proceed by induction on proofs, by focusing on the last rule.

(A) exchange rule: for simplicity we formulate the exchange rule as $\frac{\vdash \gamma, \delta}{\vdash \delta, \gamma}$. Suppose $f \in \llbracket \gamma \rrbracket \ngtr \llbracket \delta \rrbracket$ denotes the proof of $\vdash \gamma, \delta$. Recall that there is a canonical isomorphism $\llbracket \gamma \rrbracket \not \varnothing \llbracket \delta \rrbracket \simeq \llbracket \delta \rrbracket \not \gamma \llbracket \gamma \rrbracket$. Use such an isomorphism to get the denotation of the proof of $\vdash \delta, \gamma$.

(B) constant 1 rule: we denote the proof $\frac{\vdash \mathbf{1}}{\vdash}$ by $\operatorname{id}_{\mathbb{C}}$.

(C) constant $\perp$ rule: $\frac{\vdash \Gamma}{\vdash \Gamma, \perp}$.

Suppose $f \in \llbracket \Gamma \rrbracket$ denotes the proof of $\vdash \Gamma$. Then the proof of $\vdash \Gamma, \perp$ is denoted by $f \otimes \mathrm{id}_{\mathbb{C}}$.

(To check that $f \otimes \operatorname{id}_{\mathbb{C}} \in \llbracket \Gamma \rrbracket \not \gamma \perp$, use $\llbracket \Gamma \rrbracket \not \gamma \perp=\sim\{h \otimes k: h \in \sim \llbracket \Gamma \rrbracket$ and $k \in 1\})$.

(D) axioms: we denote the proof $\overline{\vdash \gamma^{\perp}, \gamma}$. by the Hermitian $\mathrm{id}_{\llbracket \gamma \rrbracket}=$ twist (see Sect. 3). Note that, by (2) of Section 2, the map $\operatorname{id}_{\mathrm{C}}=$ twist belongs to $\sim C$ \&8 $C$ for all QCS's $C$. Hence the denotations of propositional letters can be freely chosen.

(E) cut rule: for notational simplicity we formulate the cut rule as follows:

$$
\frac{\vdash \delta, \gamma \quad \vdash \gamma^{\perp}, \epsilon}{\vdash \delta, \epsilon} .
$$

Let $f \in \llbracket \delta \rrbracket \not \gamma \llbracket \gamma \rrbracket=\sim \llbracket \delta \rrbracket \multimap \llbracket \gamma \rrbracket$ and $g \in \sim \llbracket \gamma \rrbracket \not \gamma \llbracket \epsilon \rrbracket=\llbracket \gamma \rrbracket \multimap \llbracket \epsilon \rrbracket$ be denotations of the proofs of $\vdash \delta, \gamma$ and $\vdash \gamma^{\perp}, \epsilon$ respectively. We define the denotation $k \in \llbracket \gamma \rrbracket \not \llbracket \llbracket \epsilon \rrbracket=\sim \llbracket \delta \rrbracket \multimap \llbracket \epsilon \rrbracket$ of the proof of $\vdash \delta, \epsilon$ as $g \circ f$, where $\circ$ is the composition of morphisms in the category QCS defined in Section 3.

(F) tensor rule: $\frac{\vdash \gamma, \delta_{1} \vdash \delta_{2}, \epsilon}{\vdash \gamma, \delta_{1} \otimes \delta_{2}, \epsilon}$.

Let $\llbracket \gamma \rrbracket \subseteq H(X), \llbracket \delta_{1} \rrbracket \subseteq H(Y), \llbracket \delta_{2} \rrbracket \subseteq H(V), \llbracket \epsilon \rrbracket \subseteq H(W)$. Let $f \in$ $\llbracket \gamma \rrbracket \ngtr \llbracket \delta_{1} \rrbracket=\sim \llbracket \gamma \rrbracket \multimap \llbracket \delta_{1} \rrbracket$ and $g \in \llbracket \epsilon \rrbracket \ngtr \llbracket \delta_{2} \rrbracket=\sim \llbracket \epsilon \rrbracket \multimap \llbracket \delta_{2} \rrbracket$ be the denotations of the proofs of $\vdash \gamma, \delta_{1}$ and $\vdash \delta_{2}, \epsilon$ respectively.

We shall define $k \in \llbracket \gamma \rrbracket \not\left(\llbracket \delta_{1} \rrbracket \otimes \llbracket \delta_{2} \rrbracket\right) \gamma \llbracket \epsilon \rrbracket$. Let $l \in \operatorname{hom}(H(X \otimes$ $W), H(Y \otimes V))$ with the property that

$$
l(x \otimes w)=[f] x \otimes[g] w, \quad \text { for all } x \in H(X), w \in H(W) .
$$

Since the canonical isomorphism between $(X \otimes W) \otimes(Y \otimes V)$ and $X \otimes$ $(Y \otimes V) \otimes W$ induces an isomorphism between $H((X \otimes W) \otimes(Y \otimes V))$ and $H(X \otimes(Y \otimes V) \otimes W)$, the linear map $l$ uniquely determines an element 
$k \in H(X \otimes(Y \otimes V) \otimes W)$, via the map [·]. We stipulate that $k$ is the denotation of the proof of $\vdash \gamma, \delta_{1} \otimes \delta_{2}, \epsilon$.

In order to prove that $k$ is in $\llbracket \gamma \rrbracket \not \mathcal{P}\left(\llbracket \delta_{1} \rrbracket \otimes \llbracket \delta_{2} \rrbracket\right) \ngtr \curlyvee \llbracket \epsilon \rrbracket$, we first notice that, by Proposition 3.3 and by (2) of Section 2,

$\langle k, x \otimes u \otimes w\rangle=\left\langle[l]^{-1}, x \otimes w \otimes u\right\rangle=\langle l(x \otimes w), u\rangle=\langle[f] x \otimes[g] w, u\rangle$,

for all $x \in H(X), u \in H(Y \otimes V)$ and $w \in H(W)$. If $x \in \sim \llbracket \gamma \rrbracket, u \in$ $\sim\left(\llbracket \delta_{1} \rrbracket \otimes \llbracket \delta_{2} \rrbracket\right)$ and $w \in \sim \llbracket \epsilon \rrbracket$ then $[f] x \in \llbracket \delta_{1} \rrbracket$ and $[g] w \in \llbracket \delta_{2} \rrbracket$. Hence $0 \leq\langle k, x \otimes y \otimes w\rangle \leq 1$. By Lemma 3.14, $k \in \llbracket \gamma \rrbracket \ngtr\left(\llbracket \delta_{1} \rrbracket \otimes \llbracket \delta_{2} \rrbracket\right) \ngtr \curlyvee \llbracket \epsilon \rrbracket$.

(G) 8 rule: $\frac{\vdash \gamma, \delta, \epsilon}{\vdash \gamma, \delta \not \gamma \epsilon}$.

Let $g \in(\llbracket \gamma \rrbracket \not \varnothing \llbracket \delta \rrbracket) \ngtr \llbracket \llbracket \rrbracket \rrbracket$ be the denotation of the proof of $\vdash \gamma, \delta, \epsilon$. The canonical isomorphism between $(\llbracket \gamma \rrbracket \not \varnothing \llbracket \delta \rrbracket) \not \gamma \llbracket \epsilon \rrbracket$ and $\llbracket \gamma \rrbracket \not \gamma(\llbracket \delta \rrbracket \not \gamma \llbracket \epsilon \rrbracket)$ implicitly defined in the proof of Proposition 3.10 yields at once an element $k \in \llbracket \gamma \rrbracket \ngtr \gamma(\llbracket \delta \rrbracket \ngtr \curlyvee \llbracket \epsilon \rrbracket)$. More explicitly: since $(\llbracket \gamma \rrbracket \ngtr \varnothing \llbracket \delta \rrbracket) \ngtr \curlyvee \llbracket \epsilon \rrbracket=\sim((\sim$ $\llbracket \gamma \rrbracket \otimes \sim \llbracket \delta \rrbracket) \otimes \sim \llbracket \epsilon \rrbracket)$ and $\llbracket \gamma \rrbracket \not \gamma(\llbracket \delta \rrbracket \not \varnothing \llbracket \epsilon \rrbracket)=\sim(\sim \llbracket \gamma \rrbracket \otimes(\sim \llbracket \delta \rrbracket \otimes \sim \llbracket \epsilon \rrbracket))$, we use the natural isomorphism $\alpha_{\sim \llbracket \gamma \rrbracket, \sim \llbracket \delta \rrbracket, \sim \llbracket \rrbracket}$ defined in the proof of Proposition 3.18 and we proceed as in the proof of Proposition 3.8 to get the required canonical isomorphism. Let $\beta_{\llbracket \gamma \rrbracket \llbracket \llbracket \rrbracket, \llbracket \epsilon \rrbracket}$ be such an isomor-

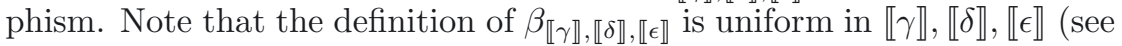
Rem. 3.19 and the proof of Prop. 3.8). Hence we may write $\beta_{X, Y, Z}$ for $\beta_{\llbracket \gamma \rrbracket, \llbracket \rrbracket \rrbracket, \llbracket \epsilon \rrbracket}$ if $\llbracket \gamma \rrbracket \subseteq H(X), \llbracket \delta \rrbracket \subseteq H(Y)$ and $\llbracket \epsilon \rrbracket \subseteq H(Z)$.

This completes the process of denotation of a proof.

So far we have not investigated the consequences of the choice of $\perp=\mathbf{1}$ as a dualizing object in the category QCS. It immediately implies that QCS is an isoMIX category, in the sense of [4], Section 6.

Recall that having $\perp=\mathbf{1}$ at the level of calculus is equivalent to having the mix and the mix $_{0}$ rules (see [1]):

$$
\frac{\vdash \Gamma \quad \vdash \Delta}{\vdash \Gamma, \Delta} \operatorname{mix} \quad-\operatorname{mix}_{0}
$$

As for derivability of mix, from $\perp=\mathbf{1}$, we get $\perp=\perp^{\perp}$ and the following derivation:

$$
\frac{\frac{\vdash \Gamma}{\vdash \Gamma, \perp} \frac{\vdash \Delta}{\vdash \perp, \Delta}}{\vdash \Gamma, \Delta} \text { cut }
$$

Hence one may add the mix and mix $x_{0}$ rule to the set of rules. In particular, we define the denotation of the empty sequent $\vdash \quad$ by letting $\llbracket \vdash \rrbracket=1$. The derivation (4) tells us at once that if $F \in \llbracket \vdash \Gamma \rrbracket$ and $G \in \llbracket \vdash \Delta \rrbracket$ are the denotations 
of the proofs of $\Gamma$ and $\Delta$ respectively, then the denotation of the proof of $\vdash \Gamma, \Delta$ obtained by application of the mix rule is just $F \otimes G$.

Similarly, the Hermitian associated to an application of mix $_{0}$ rule is id $\mathbb{C}_{\mathbb{C}}$.

When defining a denotational semantics, one is also concerned with its invariance under cut-elimination. Namely, one requires the denotation of a proof $\Pi$ being the same as the denotation of any proof $\Pi^{\prime}$ obtained from $\Pi$ by applying one step of cut-elimination. If a cut-elimination theorem holds (as is the case with MLL), an immediate consequence of invariance under cut-elimination is that if $\Pi^{\prime}$ is a cut-free transform of $\Pi$ then both have the same denotation. Indeed one asks for modular invariants (see [10]).

Since QCS is *-autonomous, from [10] we get the following:

Proposition 4.1. Let $\Pi$ be a proof in MLL and let $\Pi^{\prime}$ be obtained from $\Pi$ by applying one step of cut-elimination. Then $\Pi$ and $\Pi^{\prime}$ have the same denotation.

\section{Symmetric Tensor POWERS OF QCS's AND BOUnded EXPONENTIALS.}

The approach outlined in the previous sections cannot be extended to the infinite dimensional case, for the simple reason that the trace of an operator may not be defined. (See the discussion in [5], Sect. 17.6.1). As for the logic, this means that there is no chance of interpreting the exponentials of linear logic into a QCS's setting. Nevertheless we can incorporate in the current approach the novel bounded exponentials, whose definition is based on that of symmetric tensor power of QCS's. In turn, the latter is inspired by the symmetric tensor powers of Hilbert spaces, a central notion in quantum physics.

On the logic side, we introduce the bounded exponentials of formulas, together with their deduction rules. Basically, such rules yield a subsystem of Bounded Linear Logic ([7]). We interpret bounded exponential formulas by means of bounded exponentials of QCS's and we also extend the denotation of proofs in presence of the new rules.

In the first part of this section we recall a few standard notions, mostly for the purpose of fixing the notation.

We denote by $\otimes_{n} V$ the $n$-fold tensor power of the finite dimensional complex Hilbert space $V$. Recall that $\otimes_{n} V$ is a Hilbert space with the induced Hermitian product

$$
\left\langle\left\langle v_{1} \otimes \cdots \otimes v_{n}, w_{1} \otimes \cdots \otimes w_{n}\right\rangle\right\rangle=\prod_{i=1}^{n}\left\langle v_{i}, w_{i}\right\rangle,
$$

where $\left\langle_{-},{ }_{-}\right\rangle$is the Hermitian product on $V$. We also let $\otimes_{0} V=\mathbb{C}$. If $u \in H(V)$, we write $\otimes_{n} u$ for $\underbrace{u \otimes \cdots \otimes u}_{n \text { times }}$.

Recall that $\mathbf{1}$ is the QCS defined in the proof of Proposition 3.2. 
We define the $n$-fold tensor power $\otimes_{n} A$ of $A \subseteq H(V)$ as follows:

$\otimes_{0} A=1 ; \quad \otimes_{n} A=\sim \sim\left\{u_{1} \otimes \cdots \otimes u_{n}: u_{i} \in C\right.$ for all $\left.1 \leq i \leq n\right\}$, when $n>0$.

Clearly $\otimes_{n} A$ is a QCS and $\otimes_{n} A \subseteq H\left(\otimes_{n} V\right)$. Dually, we define

$$
\gamma_{n} A=\sim \otimes_{n}(\sim A) .
$$

Note that if $C \subseteq H(V)$ is a QCS and if we recursively define

$$
\otimes \otimes^{0} C=1 ; \quad \otimes{ }^{n+1} C=C \otimes\left(\otimes^{n} C\right),
$$

then we can prove by induction that $\otimes^{n} C$ is isomorphic to $\otimes_{n} C$, for all $n$. To prove the inductive step we assume $\otimes^{n} C \simeq \otimes_{n} C$. Then

$$
\begin{array}{rll}
\otimes_{n+1} C & \simeq \sim \sim\left\{c_{1} \otimes\left(c_{2} \otimes \cdots \otimes c_{n}\right): c_{i} \in C\right\} & =C \otimes\left\{c_{2} \otimes \cdots \otimes c_{n}: c_{i} \in C\right\} \\
& =C \otimes \sim \sim\left\{c_{2} \otimes \cdots \otimes c_{n}: c_{i} \in C\right\} & =C \otimes\left(\otimes_{n} C\right) \\
& \simeq C \otimes\left(\otimes^{n} C\right)=\otimes^{n+1} C, &
\end{array}
$$

where the rightmost equality of the first line follows from Proposition 3.16.

We denote the symmetric group on $\{1, \ldots, n\}$ by $S_{n}$ We recall that $S_{n}$ acts on the algebraic $n$-fold tensor product $\otimes_{n} H(V)$ of $H(V)$ in a natural way: let $g \in S_{n}$ and let us denote also by $g$ the isometric automorphism of $\otimes_{n} H(V)$ given by the linear extension of the mapping

$$
u_{1} \otimes u_{2} \otimes \cdots \otimes u_{n} \mapsto u_{g(1)} \otimes u_{g(2)} \otimes \cdots \otimes u_{g(n)} .
$$

As customary, we write $g u$ for $g(u)$, when $g \in S_{n}$ and $u \in H\left(\otimes_{n} V\right)$. The mapping

$$
\begin{array}{ccc}
G \times \otimes_{n} H(V) & \rightarrow & \otimes_{n} H(V) \\
(g, u) & \mapsto & g u
\end{array}
$$

yields the required action. (Note: in the literature, the action is usually defined by $(g, u) \mapsto g^{-1} u$, just for computational convenience.) For each $g \in S_{n}$, the mapping $u \mapsto g u$ is indeed a linear isometric homeomorphism of $\otimes_{n} H(V)$.

Recall that, for $n>0$, the $n$-fold symmetric tensor product of $H(V)$ is defined as follows (see [2]):

$$
\otimes_{n}^{s} H(V)=\left\{u \in \otimes_{n} H(V): g u=u \text { for all } g \in S_{n}\right\} .
$$

Proposition 5.1. $\otimes_{n}^{s} H(V)=\sim \sim\left\{\otimes_{n} v: v \in H(V)\right\}$.

Proof. Since $\otimes_{n}^{s} H(V)$ is the linear span of $\left\{\otimes_{n} v: v \in H(V)\right\}$ (see [2]), it suffices to show that $\sim\left\{\otimes_{n} v: v \in H(V)\right\}$ is a linear space. Let $c, d \in H(V)$ and $\lambda, \mu \in \mathbb{R}$. By the Bipolar Theorem, $\eta\left(\otimes_{n} e\right) \in \sim \sim\left\{\otimes_{n} v: v \in H(V)\right\}$, for all $\eta \in \mathbb{R}$ and all $e \in H(V)$. Therefore, by convexity of bipolar sets, $\frac{1}{2}\left(2 \lambda\left(\otimes_{n} c\right)+2 \mu\left(\otimes_{n} d\right)\right) \in \sim \sim$ $\left\{\otimes_{n} v: v \in H(V)\right\}$, as required. 
Inspired by Proposition 5.1, we introduce the novel notion of $n$-fold symmetric tensor product of an arbitrary set $A \subseteq H(V)$ as follows:

$$
\otimes_{0}^{s} A=1 ; \quad \otimes_{n}^{s} A=\sim \sim\left\{\otimes_{n} v: v \in A\right\}, \text { when } n>0 .
$$

Note that the set $\left\{u \in \otimes_{n} A: g u=u\right.$ for all $\left.g \in S_{n}\right\}$ is a QCS. This follows easily from the Bipolar Theorem: condition 1, 3, 4 and convexity are easily verified; as for closeness, let $\left(u_{k}\right)_{k \in \mathbf{N}}$ be a sequence in $\left\{u \in \otimes_{n} A: g u=u\right.$ for all $\left.g \in S_{n}\right\}$ such that $\left(u_{k}\right) \rightarrow u$. Then $\left(g u_{k}\right) \rightarrow g u$, namely $\left(u_{k}\right) \rightarrow g u$ for all $g \in S_{n}$. It follows that $g u=u$ for all $g \in S_{n}$, hence the conclusion. Furthermore, we have $\otimes_{n}^{s} A \subseteq\left\{u \in \otimes_{n} A: g u=u\right.$ for all $\left.g \in S_{n}\right\}$. It is an open question whether the converse inclusion holds in general. $A\}$.

It follows from Corollary 3.17 that $\otimes_{m}^{s} A \otimes \otimes_{n}^{s} A=\sim \sim\left\{\left(\otimes_{m} v\right) \otimes\left(\otimes_{n} w\right): v, w \in\right.$

Keeping in mind the definition of "\&" of QCS's given at the end of Section 3, we can now define the bounded exponentials.

Definition 5.2. The bounded exponentials $!_{n} C$ and ${ }_{n} C$ of a QCS $C$ are defined as:

$$
!_{n} C=\otimes_{n}^{s}(\mathbf{1} \& C) \quad \text { and } \quad ?_{n} C=\sim !_{n}(\sim C)
$$

respectively.

We syntactically introduce the bounded exponentials $!_{n} \gamma$ and $?_{n} \gamma=\left(!_{n}\left(\gamma^{\perp}\right)\right)^{\perp}$ of a formula $\gamma$. If $\Gamma$ is a sequence $\gamma_{1}, \ldots, \gamma_{k}$ of formulas and $\bar{n}=\left(n_{1}, \ldots, n_{k}\right)$, we write $!_{\bar{n}} \Gamma$ as a shortening for $!_{n_{1}} \gamma_{1}, \ldots, !_{n_{k}} \gamma_{k}$. Similarly with $?_{\bar{n}} \Gamma$. We let $m \bar{n}=$ $\left(m n_{1}, \ldots, m n_{k}\right)$.

The following deduction rules are available for the bounded exponentials:

$$
\begin{aligned}
& \frac{\vdash \gamma, \Delta}{\vdash ?_{1} \gamma, \Delta} \quad \text { Dereliction }(D) \quad \frac{\vdash \Delta}{\vdash ?_{0} \gamma, \Delta} \quad \text { Weakening }(W) \\
& \frac{\vdash ?_{m} \gamma, ?_{n} \gamma, \Delta}{\vdash ?_{m+n} \gamma, \Delta} \quad \text { Contraction }(C) \quad \frac{\vdash \gamma, ?_{\bar{n}} \Delta}{\vdash !_{m} \gamma, ?_{m \bar{n}} \Delta} \quad \text { Promotion }(P), \\
& \frac{\vdash ?_{0} \gamma, \Delta}{\vdash ?_{n} \gamma, \Delta} \quad \text { Deperdition }(D p)
\end{aligned}
$$

Modulo rule $C, D p$ is equivalent to the following: $\frac{\vdash ?_{m} \gamma, \Delta}{\vdash ?_{m+n} \gamma, \Delta}$.

Moreover, we have the following derived rules: $\frac{\vdash \Delta}{\vdash ?_{m} \gamma, \Delta}$ and $\frac{\vdash \gamma, \Delta}{\vdash ?_{m} \gamma, \Delta}$ for $m>0$.

We denote by B!LL the extension of MLL with the rules for the bounded exponentials. Note that B!LL is basically the intermediate system between second order Rudimentary Linear Logic and Bounded Linear Logic introduced in [7], Section 2.4, with the addition of rule $D p$. 
At first sight B!LL has some resemblance to the finitary version $\mathrm{SLL}_{f}$ of Soft Linear Logic introduced in [11], but the rules of the two systems, and their meanings, are clearly different. Nevertheless, on the semantic side, bounded exponentials are reminiscent of finitary multiplexors (see [11], Sect. 5). In spite of QCS lacking a natural structure of Cartesian category (see Sect. 3), a natural question is whether the bounded exponentials endow QCS with a finitary multiplexor category structure. An attempt of defining an endofunctor $!_{t}, t \in \mathbf{N}$, as in [11], Definition 3 leads to the obvious definition on objects, but, apparently, there is no natural and straightforward way to get a functorial extension to morphisms.

As for the denotation of the bounded exponentials, we let $\llbracket !_{n} \gamma \rrbracket=!_{n} \llbracket \gamma \rrbracket$, from which the denotation of $?_{n} \gamma$ can be immediately obtained.

Next we extend the denotation of proofs by taking into account the rules for bounded exponentials. We proceed as in Section 4, after establishing some preliminary facts.

(1) Given $f \in C_{1} \otimes \cdots \otimes C_{k} \multimap D$, we can can get $g \in\left(\mathbf{1} \& C_{1}\right) \otimes \cdots \otimes\left(\mathbf{1} \& C_{k}\right) \multimap$ $(1 \& D)$ as $[L]^{-1}$, where $L$ is any linear extension of the mapping :

$$
u_{1} \otimes \cdots \otimes u_{k} \mapsto 0 \oplus[f]\left(\pi_{2} u_{1} \iota_{2} \otimes \cdots \otimes \pi_{2} u_{k} \iota_{2}\right), \quad u_{i} \in 1 \& C_{i}, 1 \leq i \leq k .
$$

To prove that $g$ has the required property, by Lemma 3.14 it suffices to check that $\left\langle g, u_{1} \otimes \cdots \otimes u_{k} \otimes w\right\rangle \in[0,1]$, for $u_{i} \in\left(\mathbf{1} \& C_{i}\right)$ and $w \in \sim(\mathbf{1} \& D)$. The latter is equivalent to $\left\langle L\left(u_{1} \otimes \cdots \otimes u_{k}\right), w\right\rangle \in[0,1]$, which is satisfied by definition of $L$.

(2) Let $A \subseteq H(X), B \subseteq H(Y)$. Let $T: H(X) \rightarrow H(Y)$ be a linear map such that $T(A) \subseteq B$. Assuming $0 \in A$, we can define a linear map $S$ such that $S(\sim \sim A) \subseteq \sim \sim B$ by letting $S=T \circ L$, where $L: H(X) \rightarrow H(X)$ is a an arbitrarily chosen linear map such that $L(\sim \sim A) \subseteq A$. Hence $[S]^{-1} \in(\sim \sim A) \multimap(\sim \sim B)$.

Indeed, whenever we will appeal to this fact in the sequel, we will always be able to pick $0 \neq L$. We leave the reader to verify it.

Now we extend the denotation of proofs by including the rules for the bounded exponentials.

(D) It suffices to show how to associate to $f \in(C \multimap D)$ an element $g \in(C \multimap$ $1 \oplus D)$, where $C, D$ are arbitrary QCS's. We let $[g]: v \mapsto 0 \oplus[f](v)$. Clearly $[g](v) \in \mathbf{1} \oplus D$ whenever $v \in C$.

(W) Since $\llbracket ?_{0} \gamma \rrbracket=\sim \llbracket !_{0}\left(\gamma^{\perp}\right) \rrbracket=\sim \mathbf{1}=\mathbf{1}$, we proceed as in the denotation of the rule for $\perp$.

(C) First of all note that $\otimes_{m+n}^{s} C \subseteq \otimes_{m}^{s} C \otimes \otimes_{n}^{s} C$, hence $?_{m} C$ >8 $?_{n} C \subseteq ?_{m+n} C$, for all QCS's $C$. So a map $f \in\left(D \multimap ?_{m} C \ngtr ?_{n} C\right)$ is a fortiori in $D \multimap$ $?_{m+n} C$, for all QCS's $C, D$.

(P) From $f \in \llbracket !_{\bar{n}} \Delta \rrbracket \multimap \llbracket \gamma \rrbracket$, we want to get $g \in \llbracket !_{m \bar{n}} \Delta \rrbracket \multimap \llbracket !_{m} \gamma \rrbracket$. We first appeal to fact (1) above and, from $f$, we get $h \in\left(1 \& \llbracket !_{n_{1}} \delta_{1} \rrbracket\right) \otimes \cdots \otimes$ $\left(1 \& \llbracket !_{n_{k}} \delta_{k} \rrbracket\right) \multimap(1 \& \llbracket \gamma \rrbracket)$.

Then we let $A=\left\{\left(\otimes_{m} u_{1}\right) \otimes \cdots \otimes\left(\otimes_{m} u_{k}\right): u_{i} \in\left(\mathbf{1} \& \llbracket !_{n_{i}} \delta_{i} \rrbracket\right), 1 \leq i \leq\right.$ $k\}$ and $\left.B=\left\{\otimes_{m} v: v \in 1 \& \llbracket \gamma \rrbracket\right)\right\}$. Note that, by repeatedly applying 
Corollary 3.17, we get $\sim A=\otimes_{m}^{s}\left(\mathbf{1} \& \llbracket ! n_{n_{1}} \delta_{1} \rrbracket\right) \otimes \cdots \otimes \otimes_{m}^{s}\left(\mathbf{1} \& \llbracket ! n_{n_{k}} \delta_{k} \rrbracket\right)$, namely $\sim A=\llbracket !_{m} !_{n_{1}} \delta_{1} \rrbracket \otimes \cdots \otimes \llbracket !_{m} !_{n_{k}} \delta_{k} \rrbracket$. Moreover $\sim B=\llbracket ! !_{m} \gamma \rrbracket$. Let $\bar{T}$ be any linear extension of the mapping $T: A \rightarrow B$ given by $T$ : $\left(\otimes_{m} u_{1}\right) \otimes \cdots \otimes\left(\otimes_{m} u_{k}\right) \mapsto \otimes_{m}[h]\left(u_{1} \otimes \cdots \otimes u_{k}\right)$. Then, by fact $(2)$ above, we get some $\bar{h} \in\left(\llbracket ! !_{m} !_{n_{1}} \delta_{1} \rrbracket \otimes \cdots \otimes \llbracket !_{m} !_{n_{k}} \delta_{k} \rrbracket \multimap \llbracket ! m \gamma \rrbracket\right.$. Further applications of (2) yield $h_{i} \in \llbracket ! m_{n_{i}} \delta_{i} \rrbracket \multimap \llbracket ! m_{m} !_{n_{i}} \delta_{i} \rrbracket$, for all $1 \leq i \leq k$ (begin with $\otimes_{m n} u_{i} \mapsto \otimes_{m}\left(0 \oplus\left(\otimes_{n} u_{i}\right)\right)$, for $\left.u_{i} \in \mathbf{1} \& \llbracket \delta_{i} \rrbracket\right)$. Finally, we let $g=\bar{h}\left(h_{1} \otimes\right.$ $\left.\cdots \otimes h_{k}\right)$.

$(D p)$ Given $f \in \mathbf{1} \multimap C$, we define $g \in\left(\sim{ }_{n} D\right) \multimap C$. Let $L$ be a linear extension of the mapping defined by

$$
\otimes_{n} u \mapsto[f]\left(\pi_{1} u \iota_{1}\right), \quad u \in 1 \&(\sim D) .
$$

Since $\sim \sim\left\{\otimes_{n} u: u \in 1 \&(\sim D)\right\}=\sim{ }_{n} D$, from fact $(2)$ we get a linear mapping $S$ such that $S\left(\sim{ }_{n} D\right) \subseteq C$. Finally, we let $g=[S]^{-1}$.

\section{Normalization in B!LL}

In this section we outline a normalization procedure to show that B!LL has a good proof-theoretic behaviour. We also show that B!LL is well-behaved as far as the computational complexity of the normalization process is concerned. The procedure will not eliminate all cuts. The reasons for such limitation can be found in [7], to which the reader is referred for all the details. Our result is a modification of of the procedure presented therein.

Clearly, complexity of the normalization procedure is a different issue from whether B!LL or, rather, its extension to second order, provides an implicit characterization of a relevant complexity class. As already mentioned in the Introduction, the latter is beyond the scope of this work. Indeed, it appears that a second order extension of B!LL has same strength as the system described in [7], Section 2.4, that the authors claim to be powerful enough to express all feasible (i.e. polytime) functions.

In what follows, we do not want to deal with the exchange rule explicitly, so we regard sequents as formal expressions $\vdash \Gamma$, where $\Gamma$ is a finite multiset of formulas. We provide some preliminary definitions, whose motivations can be found in [7]. We say that:

- an instance of the cut rule is boxed if it is above a rule $P$;

- a cut is irreducible if it is boxed or if its left premise is obtained by application of $P$ to a sequent of length at least 2 and its right premise is obtained either by $D, W, C, P$ or $D p$;

- a B!LL proof is irreducible if it contains no reducible cuts.

(Compare with [7], Sect. 4, Appendix A).

The normalization procedure will eliminate only nonboxed cuts. We define the weight $\sharp \pi$ and the cut-size $|\pi|$ of a proof $\pi$ as in [7] (see in particular Sect. 2.4). 
We regard the rule for the constant $\mathbf{1}$ as an axiom. We stipulate that the weight increases by 1 as effect of an application of the constant $\perp$ rule or of $D p$.

Weight and cut-size of a proof are always positive natural numbers.

As in [7], we define different kinds of reductions: axiom, symmetric and commutative. We do not provide their full list, but we just present a few cases. All the other cases can be easily formulated (in particular those concerning the rules for the logical constants) or essentially already appear in [7].

None of the reduction steps applies to irreducible cuts.

- Symmetric reductions.

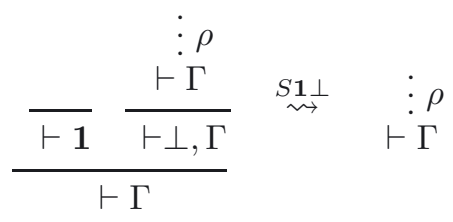

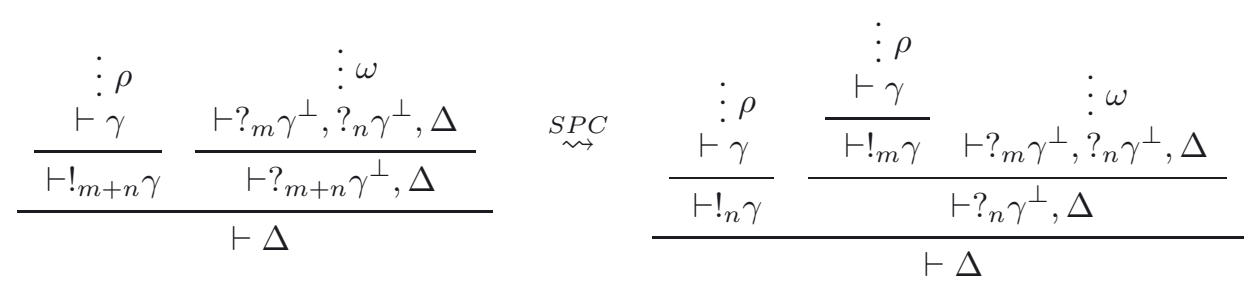

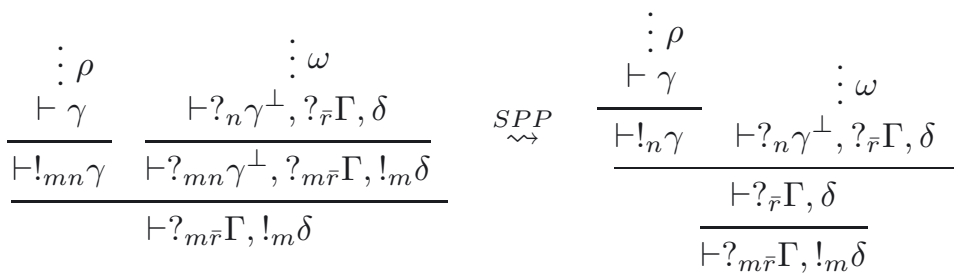

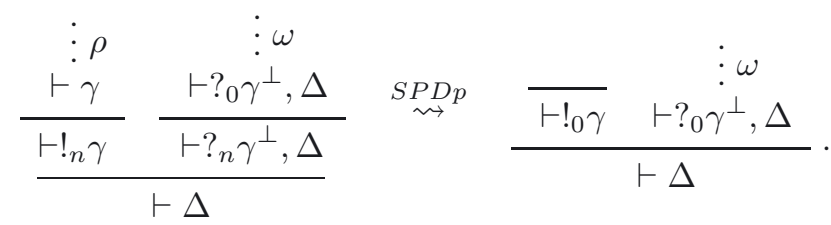

It is a matter of easy calculations to check that each symmetric reduction reduces the weight of a proof. For instance, before the $S P D p$ reduction the weight is $(\sharp \rho+1) n+1+(\sharp \omega)+1$ and decreases to $1+\sharp \omega$ after the reduction.

- Commutative reductions.

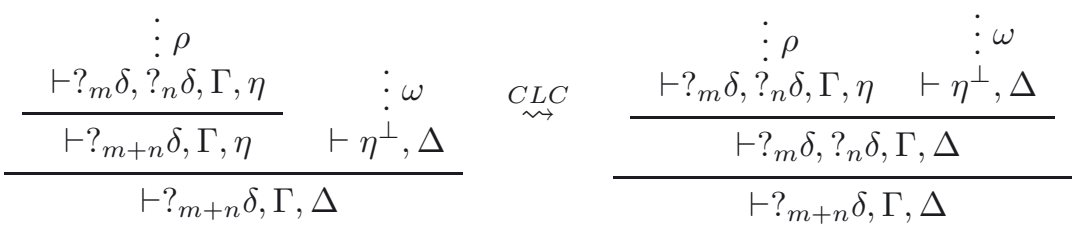



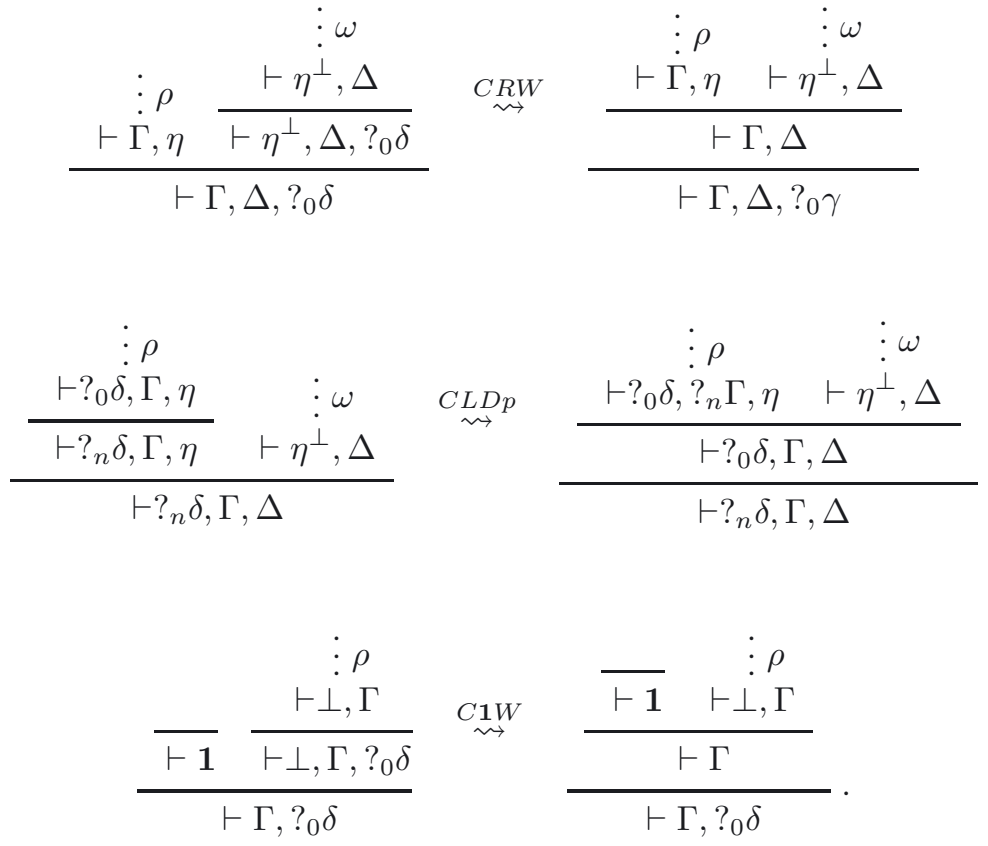

Straightforward calculations show that the commutative reductions do not increase the weight. For instance, in the case $C L D p$ above, the weights are $(\sharp \rho)+$ $(\sharp \omega)+1$ before and after the reduction step.

As in [7], Proposition 2.1, we can prove that $|\pi| \leq(\sharp \pi)^{2}$ for all B!LL proof $\pi$.

By inspecting all the cases, we can also check that the cut-size decreases after any commutative reduction. With reference to the proof trees above, we compute the cut-size before and after the reduction in a few cases:

$C L C$ - before: $|\rho|+|\omega|+\sharp \rho+\sharp \omega+4$; after: $|\rho|+|\omega|+\sharp \rho+\sharp \omega+2$.

$C L D p$ - before: $|\rho|+|\omega|+\sharp \rho+\sharp \omega+2$; after: $|\rho|+|\omega|+\sharp \rho+\sharp \omega+1$.

$C 1 W$ - before: $|\rho|+\sharp \rho+4$; after: $|\rho|+\sharp \rho+3$.

We can now summarize the content of this section in the following:

Theorem 6.1. In any B!LL proof $\pi$, any sequence of reductions on reducible cuts terminates in at most $(\sharp \pi)^{3}$ steps.

Proof. Since axiom and symmetric reductions decrease the weight, one can perform at most $\sharp \pi$ such reduction steps starting from a proof $\pi$. Moreover, between any two such reductions one can perform at most $(\sharp \pi)^{2}$ consecutive commutative reduction steps, because the commutative reductions decrease the cut-size and $|\pi| \leq(\sharp \pi)^{2}$.

Therefore any sequence of reduction steps on reducible cuts starting from $\pi$ terminates in at most $(\sharp \pi)^{3}$ steps. 


\section{REFERENCES}

[1] S. Abramsky and R. Jagadeesan, Games and full completeness for multiplicative linear logic. J. Symb. Log. 2 (1994) 543-574.

[2] J.M. Ansemil and K. Floret, The symmetric tensor product of a direct sum of locally convex spaces. Stud. Math. 129 (1998) 285-295.

[3] M. Barr, *-autonomous categories and linear logic. Math. Struct. Comp. Sci. 1 (1991) 159178.

[4] J.R.B. Cockett and R.A.G. Seely, Proof theory for full intuitionistic linear logic, bilinear logic and MIX categories. Theory and Applications of categories 3 (1997) 85-131.

[5] J.-Y. Girard, Le Point Aveugle II, Cours de logique, Vers l'imperfection. Hermann, Paris (2007).

[6] J.-Y. Girard, Truth, modality and intersubjectivity. Math. Struct. Comp. Sci. 17 (2007) 1153-1167.

[7] J.-Y. Girard, A. Scedrov and P. Scott. Bounded linear logic: a modular approach to polynomial-time computability. Theoret. Comput. Sci. 97 (1992) 1-66.

[8] S. Mac Lane, Categories for the Working Mathematician. 2nd edition Springer, Berlin (1998).

[9] R.E. Megginson, An Introduction to Banach Space Theory. Springer, Berlin (1998).

[10] P.-A. Melliès, Categorical semantics of linear logic, available at http://www.pps.jussieu. fr/ mellies/.

[11] B.F. Redmond, Multiplexor categories and models of Soft Linear Logic. Logical foundations of computer science, Lecture Notes in Comput. Sci. 4514, Springer, Berlin (2007) 472-485.

[12] P. Selinger, Towards a semantics for higher-order quantum computation. Proc. QPL (2004) $127-143$.

[13] J. Weidmann, Linear Operators in Hilbert Spaces. Springer, Berlin (1980).

Communicated by G. Longo.

Received July 7, 2010. Accepted September 14, 2010. 